\title{
FURTHER DISCUSSION ON "THE COUPLED CIRCUIT BY THE METHOD OF GENERALIZED ANGULAR VELOCITIES” BY V. BUSH
}

\author{
$\mathrm{Br}$ \\ John R. Carson \\ (American Telegraph and Telephone Company, New York)
}

Professor Bush's interesting paper appearing in the October issue of the "Proceedings of The Institute of Radio ENGINEERS" is accompanied by an appendix giving a "Summary of Wagner's Proof of Heaviside's Formula." This proof contains several fallacies which, curiously enough, are mutually destructive, so that the final formula is correct. The fallacies are, however, serious from a mathematical standpoint, and I therefore take this opportunity to point them out. It is a curious coincidence that Malcolm's proof, referred to in a footnote on page 377 , is likewise vitiated by balancing fallacies of much the same character. I might add that, inasmuch as I have not consulted Wagner's original paper, my criticisms are directed against the proof, as given by Professor Bush, only.

Professor Bush states that the infinite integral

$$
f(t)=\frac{E}{2 \pi j} \int_{-j \infty}^{j \infty} \frac{\varepsilon^{n t}}{n} d n
$$

defines a function which is zero for negative values of $t$ and $E$ for positive values of $t$. That this is incorrect is easily shown as follows: Change $t$ to $-t$ in (1), and we get

and finally

$$
\begin{aligned}
f(t) & =\frac{E}{2 \pi j} \int_{-j_{\infty}}^{j \infty} \frac{\varepsilon^{n t}}{n} d n \\
& =\frac{E}{2 \pi j} \int_{+j \infty}^{-j_{\infty}} \frac{\varepsilon^{n t}}{n} d n
\end{aligned}
$$

$$
\begin{aligned}
f(-t) & =-\frac{E}{2 \pi j} \int_{-j \infty}^{j \infty} \frac{\varepsilon^{n t}}{n} d n \\
& =-f(t)
\end{aligned}
$$

This shows that the function defined by (1) has the same absolute 
value for positive and negative values of $t$ but suffers a reversal of sign at $t=0$.

As a matter of fact the function defined by (1) is equal to $-\frac{E}{2}$ for $t<0$ and $+\frac{E}{2}$ for $t>0$. This may be readily shown in a number of ways; perhaps the easiest is to deduce it from the known value of the function

$$
\frac{2}{\pi} \int_{0}^{\infty} \frac{\sin (n t)}{n} d n
$$

which is equal to -1 for $t<0$ and +1 for $t>0$.

Professor Bush's discussion of the contour integral by which he arrives at the conclusion that the function defined by (1) is 0 for $t<0$ and $E$ for $t>0$, is defective in that it ignores the fact that the pole ( 0$)$ cuts the path of integration. When this happens no general rule can be laid down as regards the evaluation of the residue since the pole is symmetrical with respect to the two contours of integration. It may be shown, however, that for the function under consideration, $1 / 2$ the residue is to be included in each contour so that $f(t)=1 / 2 E$ for $t>0$

$$
f(t)=-1 / 2 E \text { for } t<0 \text {. }
$$

This same failure to evaluate properly the residue corresponding to the pole (0) accounts for the final formula (11) which is correct when the applied voltage is 0 for $t<0$ and $E$ for $t>0$, but is incorrect when $f(t)$ is defined by (1). The correct formula in this case is, corresponding to (11),

$$
\begin{gathered}
i=\frac{1}{2 z(0)}+\sum \frac{E}{n_{r}\left(\frac{d z}{d n}\right)_{n_{r}}} \varepsilon^{n_{r} t}, t>0 \\
i=-\frac{1}{2} \frac{E}{z(0)} \text { for } t<0 .
\end{gathered}
$$

Clearly this is the correct solution when we remember that

$$
\begin{aligned}
& f(t)=\frac{1}{2} E, t>0 \\
& f(t)=-\frac{1}{2} E, t<0
\end{aligned}
$$

The errors into which this proof falls seems to be due in part to the ambiguity which arises when the path of integration cuts one or more poles of the function. When this happens the evaluation of the residues is almost always a matter of doubt and should be justified by other methods and other considerations if possible. I would suggest in this connection that there is less chance of error if we start with the current expressed as 
a Fourier's integral; thus, corresponding to an impressed force $f(t)$, the resultant current, is

$$
i=\int_{-\infty}^{\infty} f(\lambda) \cdot d \lambda \int_{-j \infty}^{j \infty} \frac{\varepsilon^{n(t-\lambda)}}{z(n)} d n
$$

I might mention here that in the September issue of the "Physical Review," I developed and proved from dynamical considerations a general expansion theorem which holds explicity when the impressed force is an exponential function of time, and implicity for functions of arbitrary form. It is there shown that if the impressed force is $E \varepsilon^{p t}$, the resultant current (adopting Professor Bush's notation) is given by:-

$$
i=\frac{E \varepsilon^{p t}}{z(p)}-E \sum_{r}^{r} \frac{\varepsilon^{n_{r} t}}{\left(p-n_{r}\right)\left(\frac{d z}{d n}\right)_{n_{r}}} .
$$

This expression degenerates into the Heaviside formula when $p$ is put equal to zero. It enables us also to evaluate directly the transients when the impressed forces are damped or undamped sinusoidal time functions. 